\title{
Study of FPGA Based Multi-Channel Analyzer for Gamma-Ray and X-Ray Spectrometry
}

\author{
M. N. Islam, H. Akhter \\ Electronics Division, Atomic Energy Centre, Bangladesh Atomic Energy Commission, Dhaka, Bangladesh
}

\begin{abstract}
How to cite this paper: M. N. Islam | $\mathrm{H}$. Akhter "Study of FPGA Based MultiChannel Analyzer for Gamma-Ray and X-Ray Spectrometry" Published in International Journal of Trend in Scientific Research and Development (ijtsrd), ISSN: 24566470, Volume-3 | Issue-3, April 2019, pp. 61-65. http://www.ijtsrd.co $\mathrm{m} /$ papers/ijtsrd191 13.pdf

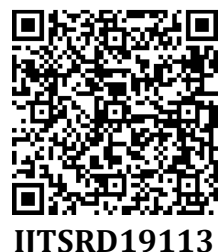

Copyright (C) 2019 by author(s) and International Journal of Trend in Scientific Research and Development Journal. This is an Open Access article distributed under the terms of the Creative Commons

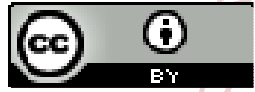
Attribution License (CC BY 4.0) (http://creativecommons.org/licenses/ by $/ 4.0$ )

\section{INTRODUCTION}

In the last decade, a great effort has been devoted to the development of instruments and systems based on X-and $\gamma$ ray spectroscopy and many of them are already operating in several research labs and also in technological sites. In fact, $\mathrm{X}$ - and $\gamma$-ray spectroscopy is a powerful diagnostic tool in several fields both in fundamental research and industrial applications, such as elemental mapping of sample composition, crystallographic determination of the solid structure, medical diagnostic, environmental monitoring, works of art investigation and nuclear structure determination [1-3].

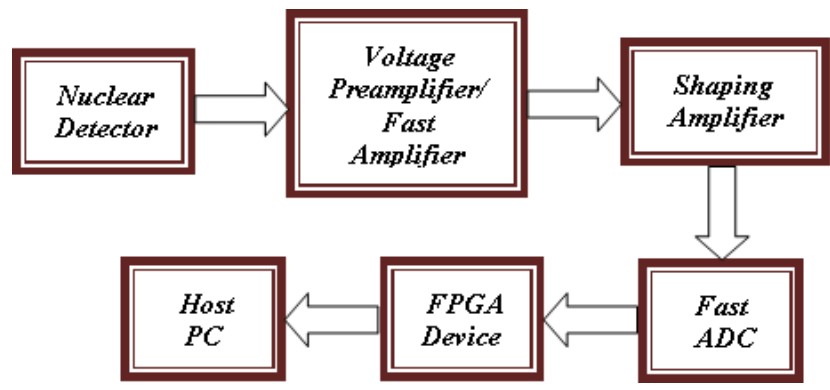

Fig.1: Block diagram shows the pulse height analyzer (PHA) based on FPGA device.

Gamma-ray spectrometry has been widely used in various environmental and natural sciences by its ability to determine the concentrations of each radionuclide of the samples, and also because of the easiness in sample preparation and measurement procedures [4]. Environmental Gamma Spectrometry is an essential tool for modeling spatio-temporal processes through coordination of data from different radiotracers. The two main advantages of environmental gamma spectrometry are: it is a nondestructive technique; and the measurement of different radiotracers is done simultaneously [5].

Therefore, the incoming pulses from the $\mathrm{NaI}(\mathrm{Tl})$ detector are fed into a fast operational amplifier. Use of a base-line stabilization block is optional and can be included as a preprocessing element. The amplified signal is converted into digital samples by means of a CMOS pipe-line ADC. Fast digital signal processing is carried out by a FPGA device. A general purpose digital signal processor (DSP) is used for pulse height spectrum generation, temporary storage, counting rate measurement as well as communication with the personal computer (PC) [6, 7]. In high-speed FPGA-based pulse-height analyzer for high resolution X-ray spectroscopy, the analog section is composed of four semi-Gaussian shaping amplifiers with two software-selectable shaping times were chosen to perform either high rate or best resolution XRF measurements with state-of-the-art multielement silicon drift detector. The pulses amplitudes are caught by four large bandwidth peak-stretchers whose outputs are multiplexed into a single analog-to-digital 
converter (ADC). A field programmable gate array (FPGA) controls the whole process and stores the four spectra in the on-chip RAM. The system interfaces the host PC by means of the enhanced parallel port with a custom made control software for data visualization and analysis as in a conventional MCA system [8].To effectively perform radiation measurements for e-Learning type exercises on a nuclear reactor, a new, compact and inexpensive modules for a pulse height analyzer (PHA) on the basis of a FPGA has been developed. Pulse signals from radiation detectors were digitized by a free-running ADC and their digitized data were distributed in parallel to four PHA modules in the FPGA. Each user independently set measurement conditions on his own PHA module. The new PHA modules were directly connected to the Internet via local area network and were successfully used for the neutron and gamma ray measurement for the e-Learning type exercises on the Kinki University reactor [9]. The current research motivated to the recent trend and development in FPGA based nuclear instruments and systems. A comprehensive study of the development of FPGA Based Multi-channel Analyzer for Gamma-ray and X-ray spectrometry has been presented.

\section{Methodology}

The main concept in the realization of the system is that the entire signal processing and pulse height analysis has to be accomplished digitally. Although the system might have some analog parts as such a fast amplifier and a base line restorer, which are placed between the detector and the ADC. The base line is not constant in the system due to the changes of temperature and counting rate, a simple circuit for base-line stabilization has been used. The central point and the most critical part of the system because of the requirement for high frequency implementation is the digital pulse processor $[10,11]$.

In accordance with the fig.2, the fast amplifier (AD8041 by Analog Devices) is used to adjust the signal from the NaI (Tl) scintillation detector to 8-bit ADC (AD9057 by Analog Devices, operating frequency of $60 \mathrm{MHz}$ ) input. Thereafter, the pulse processor FPGA (XC4008E-3 by Xilinx, operating frequency of $60 \mathrm{MHz}$ ) performs low-pass filtering, digital pulse clipping, detection of pile-up and integration of digital samples in order to determine the pulse amplitude. Only those pulses with amplitude above the threshold level are registered. The pile-up detector permits writing the result of integration of data into the output processor register and signals to the DSP (TMS320C50 by Texas Instruments) that the integration result is ready to be read in case when no pulse pile-up is detected. The other functions like energy spectrum generation and communication with the PC which donot require such a high frequency for their processing are implemented in software by using a general purpose DSP. The personal computer (PC) program was developed by use of software package for virtual instrumentation (LabWindows/CVI by National Instruments). This program enables the transfer of energy spectrum blocks from the FPGA to the PC as well as display and storage of the spectrum. Using this program, the user can change operating parameters of the pulse processor such as threshold level, level of the width of the pulses in the pile-up detection block Tp; multiplication coefficients $\mathrm{k}$ and time delay $\mathrm{n} 1 .[7,12]$.

FPGA Signal processor

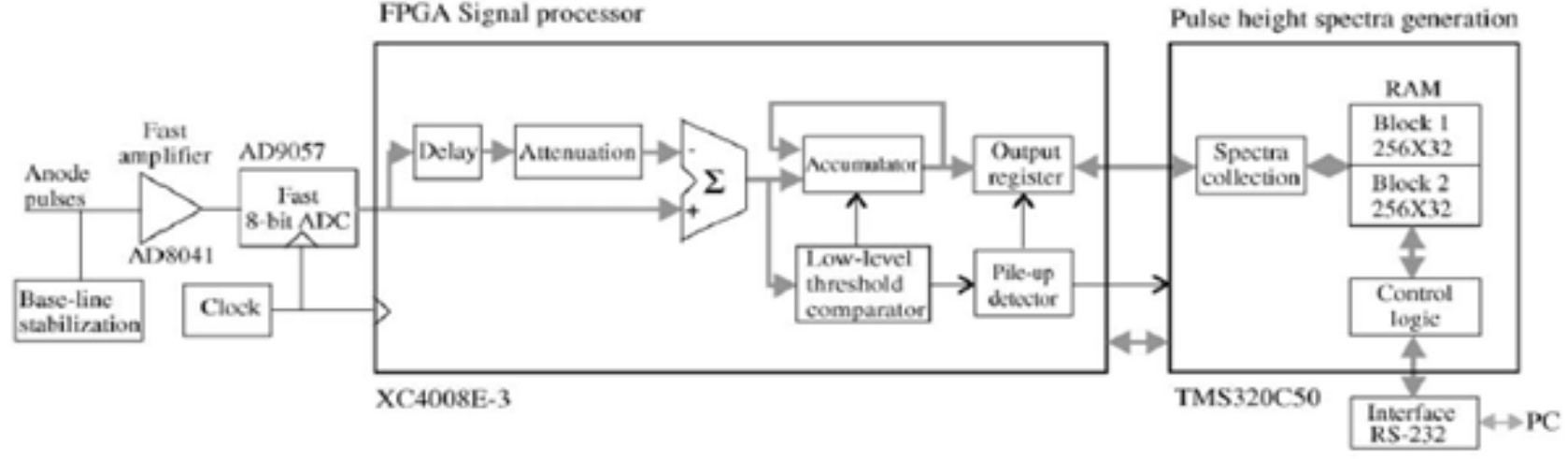

Fig.2: Block diagram of the digital pulse processing spectrometer based on FPGA device.

The signals coming from a multielement silicon drift detector are amplified by four voltage preamplifiers (VPA), each coupled to a detector channel as shown in fig.3. The preamplifier signal is fed to an analog section constituted by a custom-made 4channel shaping amplifier with digitally selectable shaping time constant of $150 \mathrm{~ns}$ for fast scanning analyses and $450 \mathrm{~ns}$ for high energy resolution elemental mapping, fast trigger signal ( 30 ns shaping time for pile-up rejection), and 4-channels peakstretcher. The analog board is a four layer PCB with SMD components housed on top of the digital board. Thanks to the fact that the AD8023 is a triple operational amplifier in a SOIC-14 package a compact layout is obtained. A single channel (shaper plus peak-stretcher) is 10 -cm-long and 3-cm-wide. The shaper needs a dual power supply ( $\pm 6 \mathrm{~V})$ and the overall dissipated power for the four channels is 3.6 W. The stretched signals are multiplexed to a AD9220 ADC by Analog Devices (12-bit data word, 10 MS/s maximum sampling frequency $10 \mathrm{MHz}$ ) that provides the conversion of the peak amplitude into a digital word. A discriminator (DISCR_S) per shaping line allows the A/D conversion only for signals higher than a minimum threshold. A discriminator follows also the fast shaping line (DISCR_F) to provide a squared signal to the pile-up rejection logic implemented in the FPGA. The core of the digital section is the FPGA that houses the RAM needed for the histogramming memory and the logic to control data acquisition, pile-up rejection and data transfer to the host PC. A dedicated control software was developed in $\mathrm{C}++$ and runs on Windows 9x, 2000, Xp. The software can manage up to 12 FPGA s boards, with 4 channels for every board $[8,13]$. 


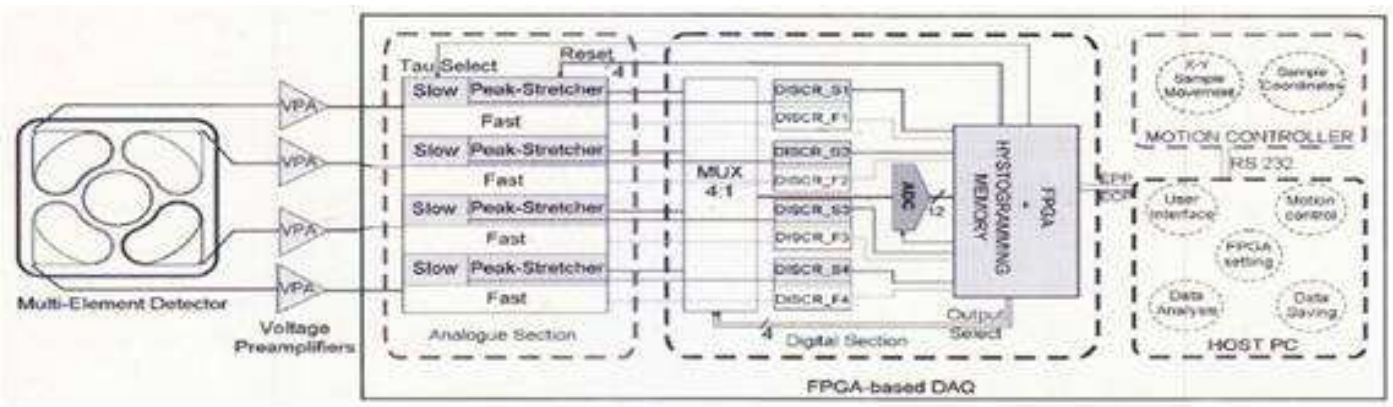

Fig.3: Scheme of principle of the FPGA-based DAQ coupled to a monolithic multianode detector.

Fig.4 shows a schematic drawing of an e-Learning system supporting exercises on a training nuclear reactor. Four PHA modular circuits for the radiation measurement were constructed in a FPGA. The radiation measurement circuit with the FPGA (FPGA circuit) is set near a training nuclear reactor (a local site), and it is interconnected to four personal computers (PCs) in a lecture room (a remote site) via the Internet. Pulse signals from a radiation detector like a NaI(Tl) scintillation counter or a BF3 proportional counter are amplified by a pre-amplifier and a shaping amplifier fed to a free-running ADC (AD9225, Analog Devices; 12 bits, 20MSPS). The pulse-shaped signals are fed into the FPGA (Cyclone EP1C3, ALTERA Corp.) circuit and are parallel processed with the four independent PHA modules and Ethernet Controller) . The four PHA modules work independently in the FPGA. Pulse signals from a BF3 or NaI(Tl) detector followed by the amplifiers are fed to a free-running ADC and a stream of digitized data is fed in parallel into the four PHA modules. In a remote site, students have access to the PHA modules through their PCs connected to the Internet. Each student can measure neutrons or gamma-rays with each assigned PHA modules on a nuclear reactor exercise. The user interface was developed by use of LabVIEW (National Instruments Corp.). There are several buttons and switches for the setting of the conditions of the PHA module [9].

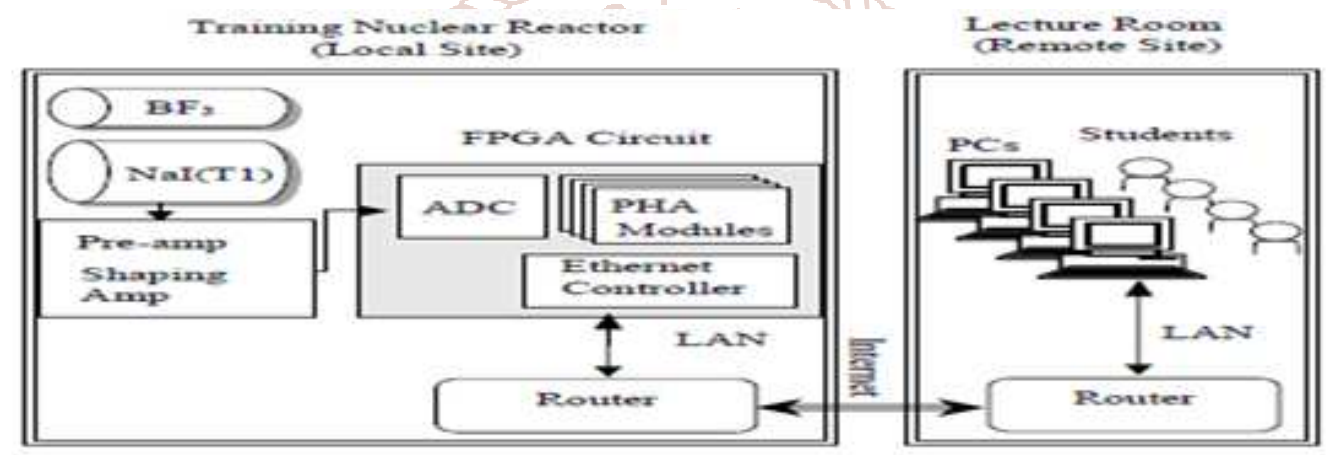

Fig.4: Schematic drawing of e-Learning system supporting exercises on training nuclear reactor.

\section{Results and Discussion}

Performance of the digital pulse processing spectrometer based on FPGA device can be roughly evaluated through the energy spectroscopy for a standard radiation source. In this context, fig. 5 shows the spectrum of ${ }^{232} \mathrm{Th}$ in the presence of ${ }^{137} \mathrm{Cs}$, fig. 6 describes energy spectrum of the fluorescence photons of a pure Cu sample irradiated with a broadband X-ray beam from a Wanode X-ray generator and fig.7 depicts an example of measured results of the photon energy spectrum for an ${ }^{241} \mathrm{Am}$ standard source.

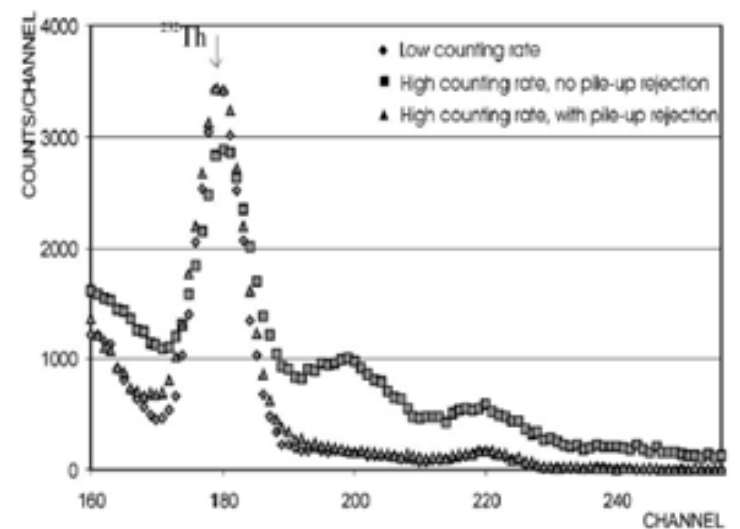

Fig.5: ( $\Delta$ ) The spectrum of ${ }^{232} \mathrm{Th}$ in the presence of ${ }^{137} \mathrm{Cs}$ at high counting rates of $400000 \mathrm{p} / \mathrm{s}$, when pulse shortening is applied and pulse pile-up detected and eliminated. (ם) Spectrum of ${ }^{232}$ Th in the presence of

${ }^{137} \mathrm{Cs}$ at high counting rates of $400000 \mathrm{p} / \mathrm{s}$ without pileup reduction by means of pulse shortening and without detection and elimination of pulse pile-up. ( ) Spectrum of ${ }^{232} \mathrm{Th}$ at low counting rates. 


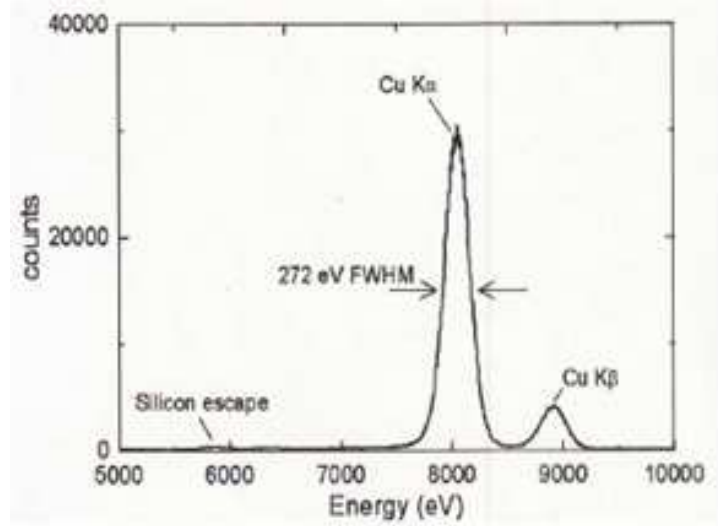

Fig.6:Energy spectrum of the fluorescence photons of a pure $\mathrm{Cu}$ sample irradiated with a broadband X-ray beam from a $\mathrm{W}$-anode $\mathrm{X}$-ray generator. The photons were detected with a 12-element monolithic matrix of silicon drift detectors.

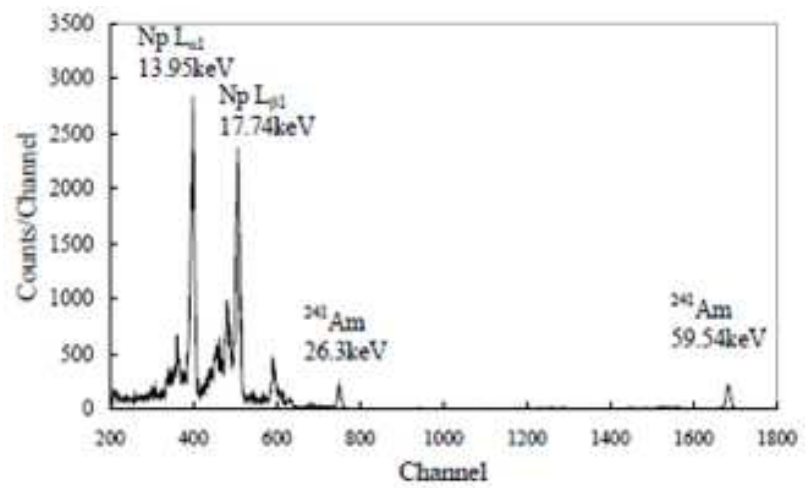

Fig.7: Measured photon energy spectrum for ${ }^{241} \mathrm{Am}$ standard source.

The performance evaluation at high counting rates was done using a low activity ${ }^{232} \mathrm{Th}$ source in the presence of ${ }^{137} \mathrm{Cs}$ of very high activity. The total counting rate in this experiment was $400000 \mathrm{~s}^{-1}$. The spectrum obtained is marked by the symbol $(\boldsymbol{\Delta})$ in fig. 5 . In order to estimate the efficiency of the applied algorithm of digital signal processing the experiment was repeated, with the system reconfigured in order to operate without pulse shortening and without pile-up detection. The energy spectrum obtained in this condition is

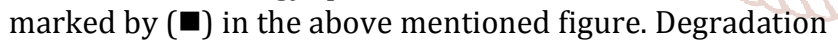
due to pulse pile-up is clearly visible. In the same figure, the symbol $(\bullet)$ mark ${ }^{232}$ Th spectrum measured at low counting rates when the effects of pile-up can be neglected [7].

Detector channels of a monolithic 12-element silicon drift detector [5] (only two channels were active in this experiment, but the data transfer to the host PC was from all 4 channels) and the fluorescence photons from a pure $\mathrm{Cu}$ sample were acquired. The $\mathrm{Cu}$ sample was illuminated by a $\mathrm{W}$ anode X-ray generator with variable anode current. Fig.6 shows a sample spectrum at an anode current of $40 \mu \mathrm{A}$ corresponding to an incident photon rate of about $15 \mathrm{KHz}$. The achieved energy resolution was limited by the detector system in the present experiment [8].

Performance of the PHA modules can be roughly evaluated through the energy spectroscopy for a standard radiation source. Fig.7 shows an example of measured results of the photon energy spectrum for an ${ }^{241} \mathrm{Am}$ standard source. The measurement was performed with a Si-PIN detector (XR100T, AMPTEK) followed by a shaping amplifier (PX2T, AMPTEK) and the present PHA modules. The position of the peaks and the values of the energy resolution for the peaks agreed well with those measured with the same detector and a commercially available multi-channel analyzer (MCA8000A, AMPTEK). Another performance test for the PHA modules was done with a pulse generator [9].

For system optimization, a simple circuit for base-line stabilization has been used in the digital pulse processing spectrometer based on FPGA device. When the pile-uprejection function is activated, the piled up pulses are completely rejected (except the ones that arrive with a time distance shorter than the time duration of the "fasf' signal used for pile-up rejection) thus cleaning up the acquired spectrum. Two essential digital circuits were designed for the triggering and peak-detecting for input pulses. The two circuits were formed in the FPGA for the pulse height analysis of a stream of digitized data. These trigger and peak detection circuits are effective for the saving of the resource of the FPGA.

\section{Conclusion}

Study of FPGA based multi-channel analyzer (MCA) for gamma-ray and $\mathrm{x}$-ray spectroscopy has been presented in this research. At first, study of digital gamma-ray spectroscopy based on FPGA technology, high-speed FPGAbased pulse-height analyzer (PHA) for high resolution x-ray spectroscopy, study about development of compact pulse height analyzer (PHA) modules based on FPGA for e-learning type exercises on nuclear reactor has been presented. Thereafter, syntheses of the system components, system performance evaluation and optimization have been provided. The energy spectrums of a digital spectrometer system for various standard sources by different detectors have been presented as well. 


\section{Acknowledgement}

The authors wish to express deep gratitude to Engr. M. Ali Zulquarnain, Chairman, Engr. Md. Monzurul Haque, Member (Engineering) and Dr. Dilip Kumar Saha, Member (Physical Science), Bangladesh Atomic Energy Commission for their support and cooperation in the research.

\section{References}

[1] C. Giacovazzo, H. L. Monaco, D. Viterbo, F. Scordari, G. Gilli, G. Zanotti and M. Catti, Fundamentals of Crystallography, Oxford Univ. press, London, U.K. 1992.

[2] R. Tertian and F. Claisse, Principles of Quantitative XRay Fluorescence Analysis, Heyden, London, U.K. 1982.

[3] T. Gog, P. M. Len, G. Materlik, D. Bahr, C. S. Fadley, and C. Sanchez-Hanke, Multiple energy x-ray holography: atomic images of hematite $\left(\mathrm{F}_{2} \mathrm{O}_{3}\right)$, Physics Revision Letter, vol. 76, no. 17, PP.3132-3135, April 1996.

[4] Jeong Hee Han and Jeong-Heon Choi, Broad Energy HPGe Gamma Spectrometry for Dose Rate Estimation for Trapped Charge Dating, Journal of Analytical Science \& Technology, Vol. 1, Issue 2, PP. 98 -108, 2010.

[5] F. J. Hernandez "Optimization of environmental gamma spectrometry using Monte Carlo methods" Ph.D. thesis, Uppsala University, Sweden. 2002.

[6] Xilinx, The Programmable Logic Company, San Jose, CA, • [13] USA, www. xilinx. com.

[7] Miodrag Bolic and Vujo Drndarevic, Digital gamma-ray spectroscopy based on FPGA technology, Nuclear Instruments and Methods in Physics Research A, Vol. 482, PP.761-766, 2002.

[8] S. Buzzetti, M. Capou, C. Guazzoni A. Longoni, R. Mariani, and S. Moser, High-Speed FPGA-Based Pulse-
Height Analyzer for High Resolution X-Ray Spectroscopy, IEEE Transactions on Nuclear Science, Vol. 52, No. 4, August 2005.

[9] Yohei IHARA, Wataru KADA, Fuminobu SATO,Toshiyuki IIDA,Junji YAMAMOTO, Sumasu YAMADA, Tetsuo HORIGUCHI, and Kengo HASHIMOTO, Development of Compact Pulse Height Analyzer Modules Based on FPGA for E-Learning Type Exercises on Nuclear Reactor, Progress in NUCLEAR SCIENCE and TECHNOLOGY, Vol. 1, p.244-247,2011.

[10] M. Al-Haddad, C. Lin, W. Miller and R. Berliner, Development and testing of a flash analog-to-digital converter based system for pulse shape discrimination of nuclear radiation pulses, IEEE Transactions on Nuclear Science, Volume: 41, Issue: 5, 1765 - 1769, Oct 1994.

[11] G. Ripamonti, A. Pullia, A. Garaci, Digital vs. analogue spectroscopy: a comparative analysis, IEEE Instrumentation and Measurement Technology Conference, St. Paul, Minnesota, May 18-21, Volume 1, 666-669, 1998.

[12] www.natinst.com, National Instrument Corporation, LabWindows/CVI-Visual Programming for Instrumentation, Ver. 3.1, Austin, TX, USA, 1995.

[13] A. Longoni, C. Fiorini, C. Guazzoni, A. Gianoncelli, L. Striider, H. Soltau, P. Lechner, A. Bjeoumikhov, J. Schmalz, N. Langhoff, and R. Wedell, A new XRF spectrometer based on a ring-shaped multi-element silicon drift detector and on X-ray capillary optics, IEEE Trans. Nucl. Sci., vol. 49, no. 3, pp. 100 1-1 005, Jun. 2002. 